\title{
Electrochemical Quartz Crystal Microbalance Studies of Electron Addition at Nanocrystalline Tin Oxide/Water and Zinc Oxide/Water Interfaces: Evidence for Band-Edge-Determining Proton Uptake
}

\author{
Buford I. Lemon and Joseph T. Hupp* \\ Department of Chemistry, Northwestern University, Evanston, Illinois 60208 \\ Received: June 17, 1996; In Final Form: January 20, 1997 ${ }^{\otimes}$
}

\begin{abstract}
Electrochemical quartz crystal microbalance (EQCM) measurements provide compelling evidence for chargecompensating cation uptake by nanocrystalline $\mathrm{SnO}_{2}$ and $\mathrm{ZnO}$ electrodes during electron addition. Comparative light water/heavy water measurements establish that the adsorbed or intercalated ions are protons or deuterons. Additional studies as a function of $\mathrm{pH}$ implicate water, rather than hydronium ions, as the proton source. The new results, when combined with previous results for titanium dioxide in nonaqueous electrolytes, suggest that charge-compensating cation intercalation is a general mode of reactivity for metal oxide semiconductors. Finally, the new observations raise significant fundamental questions concerning (1) chemical control of band energetics, (2) possible band-edge-unpinning phenomena, and (3) relationships between band edge energies and driving forces for isolated electron transfer reactions.
\end{abstract}

\section{Introduction}

$\mathrm{N}$-type nanocrystalline semiconductors based on metal oxide materials have attracted considerable attention from condensedphase chemists because of their unusual electrochemical ${ }^{1-3}$ and photochemical properties ${ }^{4}$ and the convenience with which they can be assembled in high-area thin-film ${ }^{5}$ or colloidal form. ${ }^{6}$ Valence band properties ${ }^{7}$ have been exploited in photocatalytic waste remediation, ${ }^{8}$ photochemical synthesis ${ }^{9}$ and photoelectrochemical energy conversion schemes. ${ }^{10}$ Conduction band properties, ${ }^{7}$ on the other hand, have been exploited in dye sensitization (electrochemical photovoltaic and photographic) schemes ${ }^{11}$ and in fundamental studies of fast interfacial charge transfer processes. ${ }^{12} \mathrm{~N}$-type metal oxide semiconductors also are of intrinsic interest because of their typically diode-like interfacial electrochemical behavior: In the absence of midgap surface states, current flow from a dark semiconductor electrode to a redox-active solution species is usually small except under conditions where conduction band electrons have become energetically accessible at the electrode/solution interface. ${ }^{13,14}$

For N-type semiconductors, including metal oxides, reactive electrons ${ }^{14}$ can be supplied electrochemically by externally biasing at potentials more negative than the conduction band edge $E_{\mathrm{CB}}$. They also can be supplied photochemically-either by bandgap excitation and electron/hole pair generation, followed by hole scavenging, or by dye injection. Several recent studies of metal oxide semiconductors in nanocrystalline form have shown that electron addition via both schemes can be observed optically and that the potential for the onset of addition changes, as expected, with environment-induced changes in $E_{\mathrm{CB}}$ (most notably, Nernstian shifts in $E_{\mathrm{CB}}$ with solution $\mathrm{pH}$ ). ${ }^{1 \mathrm{a}, \mathrm{e}, 3,15}$

We recently showed that electron addition to the nanocrystalline titanium dioxide/nonaqueous electrolyte interface is accompanied quantitatively by the intercalation of chargecompensating alkali metal cations or by adsorption of larger molecular cations. ${ }^{16,17}$ Here we report that the phenomenon is more general: Electron addition at nanocrystalline zinc oxide/ water and tin oxide/water interfaces is similarly accompanied by uptake of charge-compensating cations. Electrochemical

\footnotetext{
${ }^{\otimes}$ Abstract published in Advance ACS Abstracts, March 1, 1997.
}

quartz crystal microbalance (EQCM) measurements in light and heavy water establish that the adsorbed or intercalated ions are protons or deuterons, while $\mathrm{pH}$-dependent measurements indicate that the proton source is almost certainly water rather than hydronium ion. The new observations have interesting implications in terms of field effects (bulk electrodes), band edge energetics, and electron transfer mechanisms.

\section{Experimental Section}

Ethanolic $\mathrm{ZnO}$ sols were prepared by a method similar to that of Spanhel and Anderson ${ }^{18}$ as modified by Hoyer et al. ${ }^{3 \mathrm{~b}}$ This method yields nanocrystalline semiconductor particles having diameters of roughly $5 \mathrm{~nm}$. $\mathrm{SnO}_{2}$ solutions $(3-5 \mathrm{~nm}$ particle diameter) were purchased from Alfa as $15 \%$ solutions in water. To facilitate film formation from both sols, 1 drop of surfactant solution (Triton X-100, Aldrich) was added for each $\mathrm{ml}$ of solution. Solution/surfactant mixtures were then spin coated onto the keyhole-shaped gold electrode portions $\left(A_{\mathrm{e}}=\right.$ $0.205 \mathrm{~cm}^{2}$ ) of masked quartz crystals ( $5 \mathrm{MHz}$ oscillators; ICM, Inc.), with $5 \mathrm{~min}$ of heating at $100{ }^{\circ} \mathrm{C}$ between each of several coatings. Following the final coating, films were heated for 1 $\mathrm{h}$ in air at $400{ }^{\circ} \mathrm{C}$. Films were also prepared on transparent or semitransparent substrates (microscope slides, fluorine-doped tin oxide on glass (Thin-Films Research, Westford, MA) or indium-doped tin oxide on glass (ITO; PPG Industries)). These films yielded absorption spectra that were consistent with literature reports. ${ }^{19}$

EQCM measurements were performed largely as previously described ${ }^{16}$ except that an in-house module written with Labview software (National Instruments Corp., Austin, Texas) was used for PC-based data collection and instrument control.

Electrolyte solutions consisted of appropriate buffers in either $\mathrm{H}_{2} \mathrm{O}$ (Milli-Q water purification system) or $\mathrm{D}_{2} \mathrm{O}$ (Aldrich, 99.9\%). Buffering was achieved with sodium acetate/acetic acid ( $\mathrm{pH} 4$ and 5), sodium borate/hydrochloric acid ( $\mathrm{pH} \mathrm{8),} \mathrm{or} \mathrm{sodium}$ borate with $\mathrm{NaOH}(\mathrm{pH} 10)$. Electrochemical measurements were made with a PAR 273 potentiostat in a one-compartment cell containing a platinum wire counter electrode and a saturated (sodium) calomel reference electrode (ssce). All potentials are reported versus ssce. 


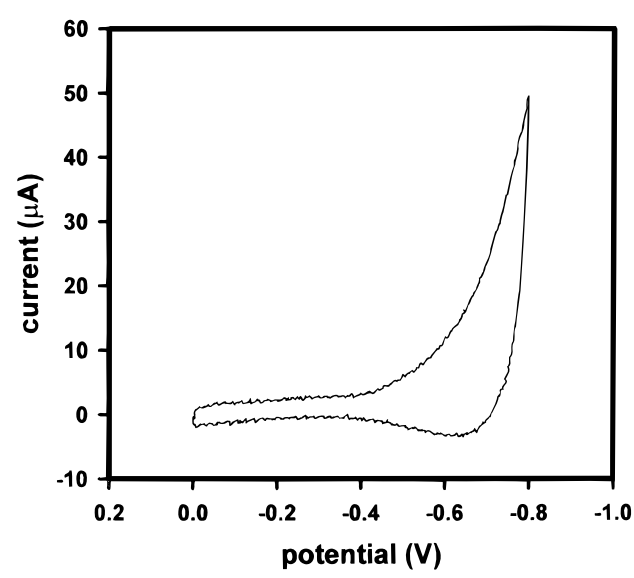

Figure 1. Cyclic voltammetry of a nanocrystalline tin oxide film at $\mathrm{pH} 10$.

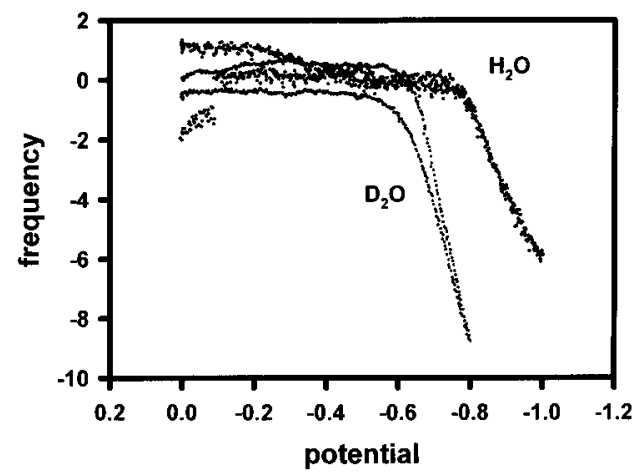

Figure 2. Oscillator frequency versus potential plot for nanocrystalline tin oxide illustrating mass uptake in $\mathrm{H}_{2} \mathrm{O}$ and $\mathrm{D}_{2} \mathrm{O}$ at potentials negative of $E_{\mathrm{CB}}$. Scan rate $=50 \mathrm{mV} / \mathrm{s}$.

\section{Results}

Tin Oxide. Nanocrystalline tin oxide was interrogated in thin-film form by electrochemical and quartz crystal microbalance methods. Figure 1 shows a voltammogram recorded at $50 \mathrm{mV} / \mathrm{s}$ in water buffered at $\mathrm{pH} 10$. At potentials positive of $-400 \mathrm{mV}$, the electrode is electronically insulating and current is absent. In an idealized description of the interface, at potentials negative of ca. $-400 \mathrm{mV}$, the Fermi level exceeds $E_{\mathrm{CB}}$ (i.e., the applied potential is more negative than $E_{\mathrm{CB}}$ ). The reversible addition of electrons is possible, as demonstrated by the passage of current. In principle, the process should yield symmetrical voltammograms. The deviations from symmetry in Figure 1 become more pronounced at lower sweep rates and in less thoroughly deoxygenated solutions. Presumably they are associated with additional current flow due to dissolved oxygen reduction and perhaps also solvent reduction.

Figure 2 shows the quartz crystal microbalance response from a tin oxide electrode under the same conditions as in the voltammetry experiment. The most notable feature is the sharp decrease in crystal oscillation frequency $f$ corresponding to an increase in film mass, $m$, beginning at $E \approx E_{\mathrm{CB}}$ (i.e., the potential where electrons begin to add to the film). From the Sauerbrey expression (eq 1), ${ }^{20}$ the absolute change in electrode mass can be estimated from the frequency change.

$$
\Delta f=\left(56.6 \mathrm{~Hz} \mathrm{~cm}^{2} \mu \mathrm{g}^{-1}\right) \Delta m / A_{\mathrm{e}}
$$

Figure 2 can then be converted to a change-in-mass versus charge plot (not shown) where the passed charge $q$ is obtained by integrating the corresponding voltammogram. From the slope of the plot, the apparent mass of the species taken up is $\sim 10$ amu. Experiments with other films yielded values ranging
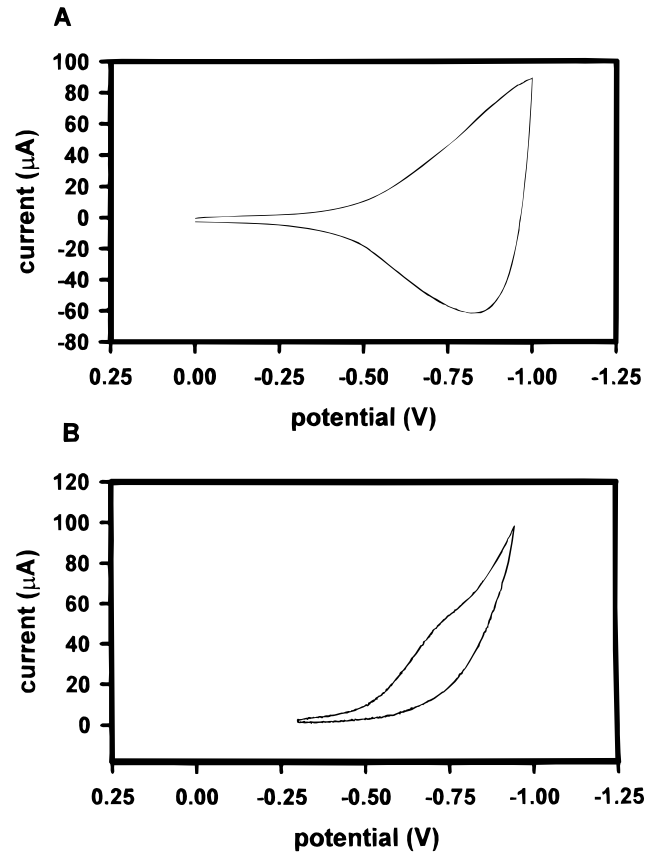

Figure 3. Cyclic voltammetry of nanocrystalline $\mathrm{ZnO}$ films at $\mathrm{pH} 8$ on substrates consisting of (a) ITO and (b) gold QCM oscillator electrode. Scan rates: $\mathrm{ITO}=100 \mathrm{mV} / \mathrm{s}$, gold $=50 \mathrm{mV} / \mathrm{s}$.

from 5 to $10 \mathrm{amu}$, suggesting the simultaneous uptake of $\mathrm{H}^{+}$ and $\mathrm{H}_{3} \mathrm{O}^{+}$. A second EQCM experiment in $\mathrm{D}_{2} \mathrm{O}$, however, yielded $\Delta f$ versus $E$ (Figure 2 ) and $\Delta f$ versus $q$ plots having almost twice the slopes of corresponding plots obtained in $\mathrm{H}_{2} \mathrm{O}$. The isotope experiments unambiguously establish the species as exclusively $\mathrm{H}^{+}$and $\mathrm{D}^{+}$. The origin of the falsely high-mass response from eq 1 is unclear but appears to be film thickness dependent. Conceivably the effect is associated in some way with the high porosity of the semiconductor film.

Similar EQCM responses were obtained at $\mathrm{pH} 5$, but with a positively shifted onset potential (as expected from the general dependence of $E_{\mathrm{CB}}$ on $\mathrm{pH}$ for metal oxide semiconductors ${ }^{13}$ ). Returning to the experiments at $\mathrm{pH} 10$, we note that the concentration of free protons or hydronium ions is necessarily vanishingly small. It follows that the source of protons, at least in high-basicity environments, is the solvent.

Zinc Oxide. Related experiments were pursued with films comprised of nanocrystalline zinc oxide. Following Weller, ${ }^{3}$ we observed reversible, symmetrical cyclic voltammetry from films prepared on flat ITO surfaces (see Figure 3A). The onset potential for nonfaradaic current flow again can be identified as approximately $E_{\mathrm{CB}}$. Nominally the same preparation on gold surfaces (coated QCM crystals) yielded much less well-defined, and much less reversible, voltammograms (Figure 3B). The differences are tentatively attributed to differences in semiconductor film thickness and effective area, with the irreversibility in Figure 3B arising from electroreduction of residual dissolved oxygen.

EQCM measurements with zinc oxide electrodes proved much more difficult than measurements with tin oxide because of the inherent chemical reactivity of $\mathrm{ZnO}$. For example, at $\mathrm{pH} 4$ we observed large, spontaneous (open circuit) mass changes due to rapid corrosion of $\mathrm{ZnO}$. Better open-circuit stability was encountered at $\mathrm{pH}$ 8. Nevertheless, significant problems with chemical irreversibility and film degradation (dissolution and/or $\mathrm{Zn}^{2+}$ migration and electroreduction) were again encountered once electrode potentials were taken negative of $E_{\mathrm{CB}}$ (ca. $-0.7 \mathrm{~V}$ at $\mathrm{pH}$ 8). Consequently, stable EQCM signals could be recorded for only a few (typically less than 10) voltammetric scans. 


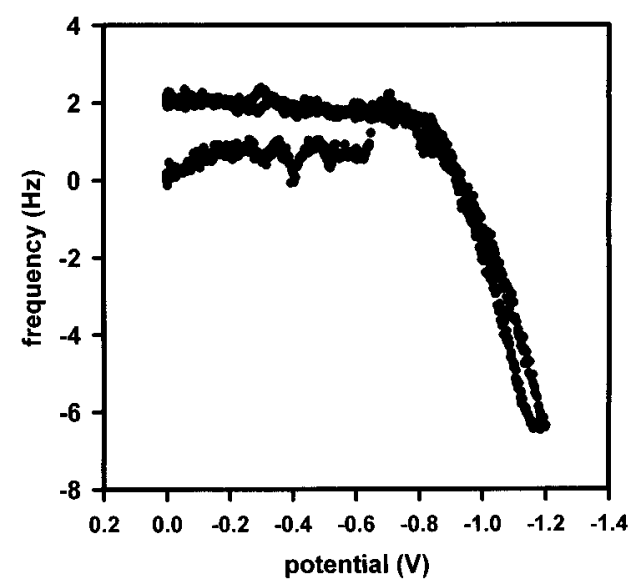

Figure 4. Oscillator frequency versus potential plot for nanocrystalline zinc oxide illustrating mass uptake at potentials negative of $E_{\mathrm{CB}}$. Scan rate $=50 \mathrm{mV} / \mathrm{s}$.

Figure 4 shows a representative "early" EQCM scan. Despite the film stability problem, the experiment clearly shows that mass increase (cation uptake) occurs at potentials more negative than $E_{\mathrm{CB}}$ (i.e., potentials where reversible electron addition can occur). We assume that the species responsible for the mass increase is again $\mathrm{H}^{+}$. Nevertheless, attempts to confirm the assumption were inconclusive. Several experiments yielded roughly 2:1 $\mathrm{D}_{2} \mathrm{O}: \mathrm{H}_{2} \mathrm{O}$ frequency change versus potential (or charge) responses; several others, however, did not. We assume that the lack of reproducibility is associated with film instability. (Note that successful implementation of the isotope experiment, for any given film, requires that film degradation be almost completely avoided over the course of successive light water/ heavy water experiments.)

\section{Discussion}

Conventional descriptions of interfacial electron addition imply charge compensation via diffuse double-layer rearrangement on the solution side of the interface. For bulk semiconductor electrodes, the descriptions also imply space charge layer formation (electrical field creation) on the semiconductor side of the interface. ${ }^{21}$ The EQCM results presented here lead to a new and qualitatively different description of interfacial energetics, at least for $\mathrm{SnO}_{2}$ /water and $\mathrm{ZnO} /$ water interfaces. For the former, the electron addition process can be written as

$\mathrm{SnO}_{2}+\mathrm{e}^{-}($external $)+\mathrm{H}^{+}($solution $) \rightarrow \mathrm{SnO}(\mathrm{OH}) \cdot \mathrm{e}^{-}{ }_{\mathrm{CB}}$

Alternatively, a more localized description would be

$$
\left.\mathrm{Sn}^{\mathrm{IV}} \mathrm{O}_{2}+\mathrm{e}^{-}(\text {external })+\mathrm{H}^{+} \text {(solution }\right) \rightarrow \mathrm{Sn}^{\mathrm{III}} \mathrm{O}(\mathrm{OH})
$$

In either case, interfacial electroneutrality is maintained by cation intercalation.

The ability of the material to achieve local electroneutrality via proton intercalation largely eliminates the need (under electron addition conditions) for compensating semiconductor space charge layer and electrolyte diffuse double-layer formation. ${ }^{22}$ The absence of significant charge separation (note that the electron and proton are intimately associated in eqs 2 and 3) should also largely eliminate the internal electric potential gradient usually assumed for space charge layer formation. On the other hand, gradients in chemical potential should still exist for both the electron and proton since intercalation presumably is a near-surface phenomenon. In view of electron/proton mobility differences, however, the chemical potential gradient should still provide a basis for current flow, albeit via diffusion rather than migration.

Returning to the issue of electric potential gradients, the elimination of internal gradients implies that the potential instead must drop primarily across the solution side of the interface, as observed for metal electrode/solution interfaces. Expressed another way, eqs 2 and 3 imply that under what have often been termed (presumably incorrectly ${ }^{1 \mathrm{~d}}$ ) accumulation conditions, the conduction band edge becomes unpinned-at least at potentials substantially negative of the nominal value for $E_{\mathrm{CB}}$. Cao et al. have discussed related optical evidence for possible band edge unpinning at the $\mathrm{TiO}_{2} /$ water interface. ${ }^{1 \mathrm{~d}}$

Equations 2 and 3 (and the corresponding expressions for zinc oxide) also suggest an interesting alternative explanation for the well-known dependence of $E_{\mathrm{CB}}$ for metal oxide semiconductors on $\mathrm{pH}$ (shifts of approximately $-60 \mathrm{mV}$ per $\mathrm{pH}$ unit). Standard explanations for energy shifts emphasize the role of open-circuit surface protonation where the resulting surface charge necessarily induces a potential perturbation. We intend to discuss the "standard" explanation and possible alternative explanations in more detail elsewhere. Briefly, however, two criticisms of the former can be made. First, if comparisons are made over a wide $\mathrm{pH}$ range, changes in $E_{\mathrm{CB}}$ can greatly exceed adsorption-induced changes in the $\zeta$ potential. Secondly, for closely related nanocrystalline titanium dioxide electrodes, Nernstian energy shifts are observable even in acidic solutions that are extremely far removed from the $\mathrm{pH}$ of zero charge (i.e., pH's where $\zeta$ potentials are invariant and all available, reactive surface oxo groups have already been protonated). ${ }^{23}$ On the other hand, the 1:1 electron:proton stoichiometric ratio and the redox or quasi-redox character of eqs 2 and 3 lead, in a straightforward fashion, to a predicted $-59 \mathrm{mV} / \mathrm{pH}$ unit shift in $E_{\mathrm{CB}}$. To expand briefly, the product state in either eq 2 or 3 is a material of fixed $\mathrm{H}^{+}$content or activity. The reactants include one component, $\mathrm{H}^{+}$(solution), of obviously variable activity. Because the conversion from reactants to product entails electron accumulation, transport or uptake, the corresponding potential $\left(E_{\mathrm{CB}}\right)$ is expected to vary as $(R T / F) \log \left\{1 / a_{\mathrm{H}+\text { (solution) }}\right\}$. We note that this Pourbaix-type interpretation, while unconventional for photoelectrode materials, is, in fact, the preferred interpretation for the electrochemistry of a number of other electrode materials, including oxides of ruthenium, ${ }^{24}$ iridium, ${ }^{25}$ manganese, ${ }^{26}$ tungsten, ${ }^{27}$ and vanadium. ${ }^{28}$

Finally, the coupled electron/proton nature of the interface energetics raises interesting questions concerning the appropriate energetics for electron transfer (ET) steps in isolation. Examples would include fast ET to and from surface-bound dye molecules. At least one recent report has suggested, based on kinetics measurements, that the dye formal potential/ $E_{\mathrm{CB}}$ energy difference may not be the correct parameter for characterization of the isolated energetics of metal oxide semiconductor (in this case, titanium dioxide) to dye electron transfer processes. ${ }^{29}$ In light of the present experiments, we hope to examine the electron transfer question for tin oxide electrodes.

\section{Conclusions}

EQCM measurements provide compelling evidence for charge-compensating cation uptake by nanocrystalline $\mathrm{SnO}_{2}$ and $\mathrm{ZnO}$ electrodes during electron addition. Solvent isotope studies show conclusively for tin oxide (but less conclusively for $\mathrm{ZnO}$ ) that the cation taken up is the proton. Additional studies as a function of solution $\mathrm{pH}$ implicate water, rather than hydronium ion, as the proton source. The new results, when combined 
with previous results for titanium dioxide in nonaqueous electrolytes, suggest that charge-compensating cation intercalation may well represent a general mode of reactivity for metal oxide semiconductors. Finally, the new observations raise interesting fundamental questions regarding (1) chemical control of band energetics, (2) possible band edge unpinning phenomena, and (3) relationships between band edge energies and driving forces for isolated electron transfer reactions.

Acknowledgment. We thank the Office of Naval Research and the DOD AASERT program for support of our research. J.T.H. also gratefully acknowledges unrestricted support from the Dreyfus Foundation (Teacher-Scholar Award, 1991-96).

\section{References and Notes}

(1) For representative studies of $\mathrm{TiO}_{2}$, see: (a) Rothenberger, G.; Fitzmaurice, D.; Grätzel, M. J. Phys. Chem. 1992, 96, 5983. (b) Redmond, G.; Fitzmaurice, D. J. Phys. Chem. 1993, 97, 1426. (c) Basame, S. B.; White, H. S. J. Phys. Chem. 1995, 99, 16430. (d) Cao, F.; Oskam, G.; Searson, P. C.; Stipkala, J. M.; Heimer, T. A.; Farzad, F.; Meyer, G. M. J. Phys. Chem. 1995, 99, 11974. (e) Enright, B.; Redmond, G.; Fitzmaurice, D. J. Phys. Chem. 1994, 98, 6195.

(2) For representative studies of $\mathrm{SnO}_{2}$, see: (a) Nas, R.; Kamat, P. V. Langmuir 1995, 11, 1777. (b) Liu, K.; Kamat, P. V. J. Electrochem. Soc. 1995, 142,835 .

(3) For representative studies of $\mathrm{ZnO}$, see: (a) Hoyer, P.; Weller, H. J. Phys. Chem. 1995, 99, 14096. (b) Hoyer, B.; Eichberger, R.; Weller, H. Ber. Bunsen-Ges. Phys. Chem. 1993, 97, 630.

(4) For reviews, see: (a) Hagfeldt, A.; Gratzel, M.; Chem. Rev. 1995, 95, 49. (b) Weller, H.; Eychmuller, A. Adv. Photochem. 1995, 20, 165.

(5) For example, see: (a) Kavan, L.; O'Regan, B.; Kay, A.; Grätzel, M. J. Electroanal. Chem. 1993, 346, 291. (b) Hotchandani, S.; Kamat, P. V. J. Electrochem. Soc. 1992, 139, 1630.

(6) For representative work, see: (a) Kormann, C.; Bahnemann, D. W.; Hoffmann, M. R. J. Phys. Chem. 1988, 92, 5196. (b) Koch, U.; Fojtik, A.; Weller, H.; Henglein, A. Chem. Phys. Lett. 1985, 122, 507.

(7) We recognize that apparent valence band and conduction band reactivity could also correspond to reactivity from shallow traps or from otherwise localized holes or electrons.

(8) For a recent review, see: Rajeshwar, K. J. Appl. Electrochem. 1995, $25,1067$.
(9) (a) Wang, C. M.; Mir, Q.-C.; Malekania, S.; Mallouk, T. E. J. Am. Chem. Soc. 1988, 110, 3710. (b) Wang, C. M.; Mallouk, T. E. J. Phys. Chem. 1990, 94, 423.

(10) For example, see: Bard, A. J. J. Phys. Chem. 1982, 86, 172.

(11) For reviews, see: (a) Meyer, G. J.; Searson, P. C. Interface 1993, 33, 115. (b) Grätzel, M. Coord. Chem. Rev. 1991, 111, 167. (c) Kamat, P. V. Chem. Rev. 1993, 93, 267.

(12) (a) Lu, H.; Preiskorn, J. N.; Hupp, J. T. J. Am. Chem. Soc. 1993, 115, 4927. (b) Yan, S. G.; Hupp, J. T. J. Phys. Chem. 1996, 100, 6867. (c) Moser, J.; Grätzel, M. Chem. Phys. 1993, 176, 493. (d) Ford, W. E.; Rodgers, M. A. J. J. Phys. Chem. 1994, 98, 3822. (e) Fessenden, R. W.; Kamat, P. V. J. Phys. Chem. 1995, 99, 12902. (f) Eichberger, R.; Willig, F. Chem. Phys. 1990, 141, 159.

(13) Bolts, J. M.; Wrighton, M. S. J. Phys. Chem. 1976, 80, 2641.

(14) For ideal semiconductor/solution interfaces, currents (albeit typically small currents) can also flow under conditions of weak depletion.

(15) Bejda, I.; Hotchandani, S.; Kamat, P. V. J. Phys. Chem. 1993, 97, 11064.

(16) Lyon, L. A.; Hupp, J. T. J. Phys. Chem. 1995, 99, 15718.

(17) See also: (a) Hagfeldt, A.; Vlachopoulos, N.; Grätzel, M. J. Electrochem. Soc. 1994, 141, L82. (b) Kavan, L.; Kratochvilová, K.; Grätzel, M. J. Electroanal. Chem. 1995, 394, 93.

(18) Spanhel, L.; Anderson, M. A. J. Am. Chem. Soc. 1991, 113, 2826

(19) (a) Bejda, I.; Hotchandani, S; Kamat, P. V. J. Phys. Chem. 1994,

98, 4133. (b) Redmond, G.; O'Keefe, A.; Burgess,C.; MacHale, C.; Fitzmaurice, D. J. Phys. Chem. 1993, 97, 11081.

(20) Sauerbrey, G. Z. Phys. 1959, 155, 206.

(21) For example, see: Morrison, S. R. The Chemical Physics of Semiconductor Surfaces; Plenum Press: New York, 1977.

(22) On the other hand, the description does allow for space charge layer formation under depletion conditions. Compelling optical evidence for depletion-based space charge layer creation within bulk titanium dioxide electrodes has recently been reported by Lantz, Baba, and Corn (J. Phys. Chem. 1993, 97, 7392).

(23) Lemon, B. I.; Lyon. L. A., unpublished experimental data.

(24) Mitchell, D.; Rand, D. A. J.; Woods, R. J. Electroanal. Chem. 1978, $89,11$.

(25) (a) Pickup, P. G.; Birss, V. I. J. Electroanal. Chem. 1988, 240, 185. (b) Burke, L. D.; Whelan, D. P. J. Electroanal. Chem. 1984, 121, 121. (c) Johnson, C. S.; Hupp, J. T. J. Electroanal. Chem. 1993, 345, 351.

(26) Burke, L. D.; Murphy, O. J. J. Electroanal. Chem. 1980, 109, 373.

(27) Natan, M. J.; Wrighton, M. S. J. Phys. Chem. 1987, 91, 648.

(28) Burke, L. D.; O’Sullivan, E. J. M. J. Electroanal. Chem. 1980, $111,383$.

(29) Yan, S. G.; Hupp, J. T. J. Phys. Chem. 1996, $100,6867$. 